\title{
Multichannel Operation of an Integrated Acousto- Optic Wavelength Routing Switch for WDM Systems
}

\author{
A. d'Alessandro, D. A. Smith, Senior Member, IEEE, and J. E. Baran
}

\begin{abstract}
Polarization independent acousto-optic tunable filters (PIAOTF's) can operate as transparent wavelength-selective crossconnects to route signals in wavelength division multiplexed optical networks. In this paper, a new low power PIAOTF is characterized as a switch in multiwavelength operation, using four equally spaced lightwave signals with wavelengths between $1546 \mathrm{~nm}$ and $1558 \mathrm{~nm}$. Interchannel interference due to sidelobe excitation is lower than $-11 \mathrm{~dB}$ for single wavelength switching and is equal to $-6 \mathrm{~dB}$ in the extreme case of simultaneous switching of all wavelength channels. Sources of interport and interchannel crosstalk for single and multiple wavelength switching are identified.
\end{abstract}

\section{INTRODUCTION}

W AVELENGTH division multiplexed (WDM) optical network architectures require optical crossconnect switches to switch and route optical signals [1]. The acoustooptic tunable filter (AOTF) is interesting because it can operate as a wavelength-selective optical crossconnect, in which optical routing is transparent in a given wavelength band, but the fiber connections can be independently set for each "color". Furthermore AOTF's have the capability, which makes them unique among equivalent optical filters/switches, of processing many optical signals simultaneously and independently [2]. The use of an AOTF as a WDM add-drop multiplexer has been demonstrated by various workers [3], [4].

In an AOTF the selection of optical channels is accomplished by means of a narrow-band resonant acousto-optic polarization conversion of light. The RF-to-optical frequency matching condition is $F_{j}=v_{\text {sound }} \Delta n / \lambda_{j}$, in which $\lambda_{j}$ is the resonant wavelength, $v_{\text {sound }}$ is the speed of sound and $\Delta n$ is the effective birefringence of the acousto-optic medium.

Since the PIAOTF is being employed in WDM systems demonstrations, a characterization of this device in multiple channel operation is required. In particular, one important concern in multiwavelength operation of AOTF's is interport and interchannel crosstalk. In this paper we discuss the results obtained from an experiment, to analyze the multiwavelength operation of a two input-two output PIAOTF, using four 4 $\mathrm{nm}$-spaced signals in the $1550 \mathrm{~nm}$ spectral window.

\section{SETUP}

Fig. 1 shows the setup arranged to perform a multiwavelength characterization of the PIAOTF sketched in the inset

Manuscript received July 26, 1993; revised November 2, 1993.

The authors are with Bellcore, NVC 3X-385, 331 Newman Springs Road, Red Bank, NJ 07701, USA

IEEE Log Number 9216608.
The lightwave sources were four single-frequency DFB lasers emitting at wavelengths $\lambda_{1}=1546 \mathrm{~nm}, \lambda_{2}=1550 \mathrm{~nm}, \lambda_{3}=$ $1554 \mathrm{~nm}$ and $\lambda_{4}=1558 \mathrm{~nm}$, used as channel standards in an all-optical demonstration network that uses PIAOTF's as WDM crossconnect switches [5]. Each laser was provided with a polarization controller (PC) to allow measurements in both TE and TM polarizations. The four infrared signals were combined by means of three optical directional couplers (DC) and were routed into the input waveguide of the filter/switch and then out to an optical spectrum analyzer. The inset of Fig. 1 is a schematic of a novel low power PIAOTF, fabricated on a 5-cm-long XY lithium niobate crystal with passive annealed proton exchanged/titanium in-diffused (APE/Ti) polarization beam splitters/combiners (PBS/C), employed to realize a polarization diversity device. The APE/Ti junction adiabatically routes TE polarized light from one optical Ti waveguide to the other parallel guide, while the orthogonal TM polarization remains in its original input waveguide. The APE/Ti approach has the potential of high extinction ratios due to the adiabatic coupling of a single state of polarization between APE branches and Ti-indiffused waveguides [6]. Other approaches include PBS/C's using pure Ti in-diffusion [7] or active electro-optically tuned PBS/C's [8]. Moreover the device was conceived as a two-input/two-output switch: if a given wavelength channel $\lambda_{j}$ is selected, optical signals of that wavelength coming from port 1 are routed to port $2^{\prime}$ and from port 2 towards $1^{\prime}$ (switch in the cross-state for $\lambda_{j}$ ). Unselected signals are routed from 1 to $1^{\prime}$ and 2 to $2^{\prime}$ (switch in bar-state). The driving circuitry consisted of four synthesizers, generating four different pure RF frequencies at the values of frequencies $F_{1}=175.89 \mathrm{MHz}, F_{2}=175.38 \mathrm{MHz}, F_{3}=174.86 \mathrm{MHz}$ and $F_{4}=174.42 \mathrm{MHz}$ for selecting the four wavelengths, respectively. The RF signals were combined, amplified and applied to the interdigital transducer of the PIAOTF. A driving power of just $11.2 \mathrm{dBm} /$ channel was required to get the maximum polarization conversion.

\section{RESULTS AND DISCUSSION}

Fig. 2 shows the photographs of the oscilloscope traces representing the light intensity transmitted at $1550 \mathrm{~nm}$ as a function of RF frequency over a ramp of $3 \mathrm{MHz}$ (equivalent to a $26 \mathrm{~nm}$ wavelength scan), centered at $175.5 \mathrm{MHz}$, both for bar-state (Fig. 2a) and for cross-state (Fig. 2b). The The interaction length of the device was $2 \mathrm{~cm}$, measured between the IDT and the acoustic absorber, yielding a FWHM of $1.6 \mathrm{~nm}$. Table I summarizes the complete set of results of the multiwavelength experiment taken for all different 


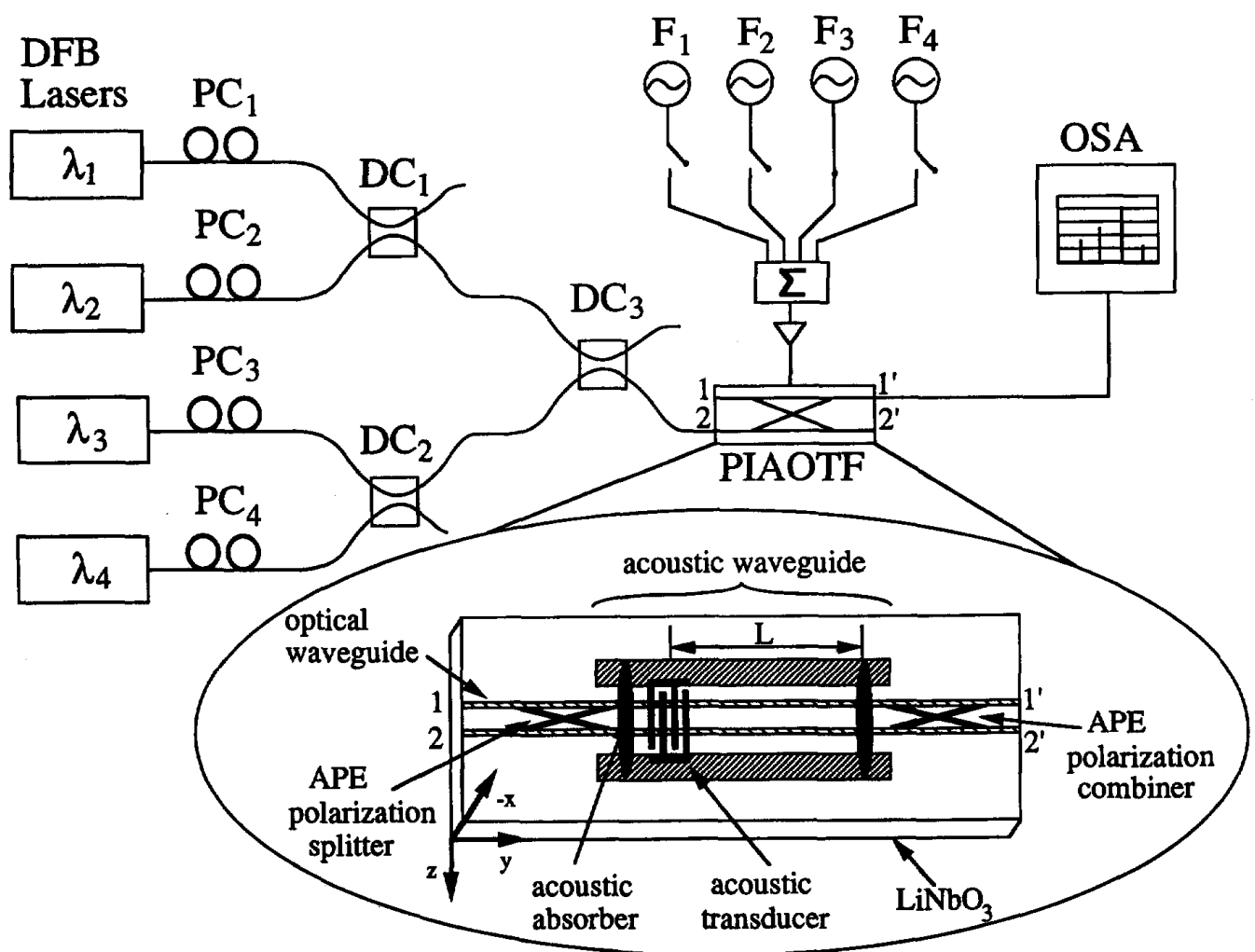

Fig. 1. Set-up of the multiwavelength experiment using four DFB diode lasers feeding four single mode fibers provided with polarization controllers (PC) and directional couplers (DC) to multiplex the four lightwave signals into a single fiber feeding the PIAOTF, whose schematic is shown in the inset.

combinations of the driving signals $(1=$ on, $0=$ off $)$, for both bar-state and cross-state, for TM-polarized input light at port 1 . The accuracy of the measurements is within $\pm 0.5 \mathrm{~dB}$, limited by power fluctuations of the lasers. During passive operation of the device (all four RF drive frequencies off, described by the nomenclature 0000 ) the light intensities detected at the output port 1' were the same and were chosen as reference levels to measure the bar-state extinction, defined as the residual relative light intensity at a certain wavelength when the corresponding resonant RF signal is applied. During single channel operation (corresponding to RF combinations $1000,0100,0010$ and 0001) the light intensities of the selected wavelengths, detected at the output port 2', were equal and were chosen as reference level to measure the extinction levels for the cross-state, defined as the relative intensity of light of non-selected channels. TE and TM performance was comparable, except for passive cross-state extinction (the 0000 state). During passive operation cross-state extinction levels were over $-24 \mathrm{~dB}$ for TM polarization, as shown in Table I, about $10 \mathrm{~dB}$ better than for TE. This difference is due to an incomplete coupling of TE light into the APE branch involved in the optical path, in this particular sample. We use the TM results in order to simplify the crosstalk analysis, since TM leakage was negligible. During single channel operation [9], extinction levels for the bar state ranged between $-11 \mathrm{~dB}$ and $-13 \mathrm{~dB}$, limited by leakage of selected (TE-polarized) light in the output polarization combiner. A profound improvement for both bar-state and cross-state extinctions can be obtained by dilating $\mathrm{AO}$ switches [10]. In dilated AO switches the polluting signal residuals are addressed to an unused optical port with only a second order coupling to used signal channels.

The values of sidelobe-related (interport) crosstalk are the cross-state extinctions of unswitched neighboring channels. In single channel operation, crosstalk values are over -16 $\mathrm{dB}$ for longer wavelength neighboring channels. Crosstalk is not better than $-8 \mathrm{~dB}$ for shorter wavelength neighboring channels, as indicated by the cross-state values for the states $1000,0100,0010$ and 0001 . This strong difference of crosstalk in the two sides of the optical spectrum is due to the sidelobe asymmetry of the transmission function of the device as clearly shown in Fig. 2 . This asymmetry can be caused by a systematic variation of effective birefringence along the optical waveguides which can be largely eliminated by improved waveguide width and Ti thickness uniformity [11], [12].

During multichannel operation another effect related to high sidelobes is the degradation of the bar-state extinction. This effect can be observed, for example, by comparing the values of bar-state extinctions when channels 3 and 4 are selected simultaneously (state 0011), with the extinctions measured by selecting channels 3 and 4 individually (states 0010 and 0001 respectively). In the state 0011 the bar-state extinction of channel 3 is $5 \mathrm{~dB}$ worse than in the state 0010 . Extinction 
TABLE I

MeAsuRements OF Relative INTENSITIES IN MultrWAVELENGTH SWITCHING BY FEEDING INPUT PORT 1 WITH TM POLARIZED LIGHT.

\begin{tabular}{cccccccccccc}
\hline \multicolumn{3}{c}{$\begin{array}{c}\text { RF excitation 1=on, } \\
0=\text { off }\end{array}$} & \multicolumn{1}{c}{ Bar state values $\left(^{*}\right)$} & \multicolumn{3}{c}{ Cross state values ${ }^{* *}$ ) } \\
\hline $\mathrm{F}_{1}$ & $\mathrm{~F}_{2}$ & $\mathrm{~F}_{3}$ & $\mathrm{~F}_{4}$ & $\lambda_{1}$ & $\lambda_{2}$ & $\lambda_{3}$ & $\lambda_{4}$ & $\lambda_{1}$ & $\lambda_{2}$ & $\lambda_{3}$ & $\lambda_{4}$ \\
\hline 0 & 0 & 0 & 0 & 0 & 0 & 0 & 0 & -27 & -24 & -24 & -25 \\
1 & 0 & 0 & 0 & -11 & 0 & 0 & 0 & 0 & -16 & -18 & -21 \\
0 & 1 & 0 & 0 & 0 & -12 & 0 & 0 & -7 & 0 & -17 & -19 \\
0 & 0 & 1 & 0 & 0 & 0 & -13 & 0 & -16 & -8 & 0 & -17 \\
0 & 0 & 0 & 1 & 0 & 0 & 0 & -11 & -16 & -13 & -7 & 0 \\
0 & 0 & 1 & 1 & 0 & 0 & -8 & -11 & -15 & -8 & 0 & 0 \\
0 & 1 & 0 & 1 & 0 & -11 & 0 & -11 & -7 & 0 & -7 & 0 \\
1 & 0 & 0 & 1 & -11 & 0 & 0 & -11 & 0 & -10 & -7 & 0 \\
0 & 1 & 1 & 0 & 0 & -11 & -12 & 0 & -7 & 0 & 0 & -15 \\
1 & 0 & 1 & 0 & -10 & 0 & -11 & 0 & 0 & -7 & 0 & -16 \\
1 & 1 & 0 & 0 & -7 & -13 & 0 & 0 & 0 & 0 & -14 & -16 \\
0 & 1 & 1 & 1 & 0 & -8 & -9 & -12 & -6 & 0 & 0 & 0 \\
1 & 0 & 1 & 1 & -10 & 0 & -10 & -10 & 0 & -6 & 0 & 0 \\
1 & 1 & 0 & 1 & -7 & -10 & 0 & -10 & 0 & 0 & -9 & 0 \\
1 & 1 & 1 & 0 & -6 & -9 & -12 & 0 & 0 & 0 & 0 & -13 \\
1 & 1 & 1 & 1 & -6 & -8 & -9 & -11 & 0 & 0 & 0 & 0 \\
\hline
\end{tabular}

(*) Values in $\mathrm{dB}$ relative to the light intensity from output port 1' when the RF signals are not active.

(**) Values in $\mathrm{dB}$ relative to the light intensity from output port 2' when the RF signals are active.

of channel 4 is not affected by the selection of channel 3 because the longer wavelength optical spectrum of the transmission function of channel 3 is sidelobe-suppressed. There is almost no deterioration of bar-state extinction when two next-nearest neighbor channels are switched, as was observed when comparing the extinctions for the state 1001 with the extinctions for 1000 and 0001 ; or when comparing the results for state 0101 with those for 0100 and 0001 . The degradation increases when 3 or 4 channels are selected: a bar-state extinction of only $-6 \mathrm{~dB}$ was measured for channel 1 in the states 1110 and 1111 . The degradation of bar-state conversion can be interpreted as the result of the overlap of competing diffraction gratings, created by the two or more acoustic waves simultaneously traveling along the acoustooptic interaction region. The overlap is due to the presence of the sidelobes that affect neighboring optical signals and overdrive the polarization conversion.

By turning on more than one RF frequency the crosstalk values in the cross-state get worse too. For example in the case 1010 , crosstalk level at wavelength 2 increases to -7 $\mathrm{dB}$ from its initial value of -16 before turning on $F_{3}$ (case 1000 ). The worse level of crosstalk is $-6 \mathrm{~dB}$, as can be read in Table I.

\section{CONCLUDING REMARKS}

The main limitation in multiwavelength operation of PIAOTF is crosstalk. In this paper we have shown that interport and interchannel crosstalk can be generated by two main sources: i) leakage through polarization beam splitters/combiners; and ii) high sidelobe levels of the transmission function. The first source of interport crosstalk can be greatly reduced both by improving fabrication of passive hybrid APE/Ti PBS/C's and by using in addition cascaded (or dilated) PIAOTF's. When two or more RF (a)

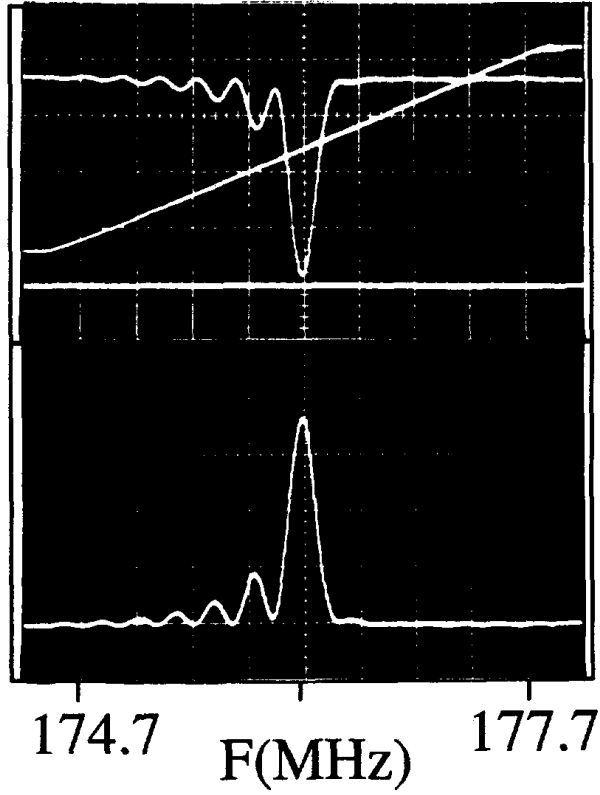

Fig. 2. Oscilloscope traces of the bar-state (a) and cross-state (b) transmission function of the PIAOTF at $1550 \mathrm{~nm}$ during a $3 \mathrm{MHz}$ sweep centered at about $175.5 \mathrm{MHz}$.

frequencies are applied, coherent crosstalk can be generated by simultaneous switching of one wavelength channel by more than one acoustic grating, each with associated imposed frequency up-or-down shift [13]. Even though dilation reduces sidelobes in the transmission function, the particular interference mechanism of coherent crosstalk determined by sidelobes cannot be eliminated by cascading more AOTF's. Low crosstalk AO switches can only be obtained suppressing sidelobes in each AO unit, by using a tapered acousto-optic interaction, achievable by a number of different techniques [14]-[17].

\section{ACKNOWLEDGMENT}

This work is supported in part by a consortium grant MDA 972-92-H-0010.

\section{REFERENCES}

[1] C. A. Brackett, "Dense wavelength division multiplexing networks: Principles and applications," IEEE J. Selected Areas in Commun., vol. 8, no. 6, p. $948,1990$.

[2] K. W. Cheung, D. A. Smith, J. E. Baran and B. L. Heffner "Multiple channel operation of an integrated acousto-optic tunable filter," Electron. Lett., vol. 25, p. 375, 1989.

[3] W. I. Way, D. A. Smith. J. J. Johnson and H. Izadpanah, "A self-routing WDM high-capacity SONET ring network," IEEE Photon. Technol. Lett., vol. 4, no. 4, p. 402, 1992.

[4] M. Fukutoku, K. Oda and H. Toba: "Wavelength-division-multiplexing add/drop multiplexer employing a novel polarisation independent acousto-optic tunable filter," Electron. Lett., vol. 29, p. 905-907, 1993

[5] C. A. Brackett, A. S. Acampora, J. Sweitzer, G. Tangonan, M. T. Smith W. Lennon, K. C. Wang and R. H. Hobbs: "A Scalable multiwavelength multihop optical network: A proposal for research on all-optical networks," J. of Lightwave Technol., vol. 11, p. 736-753, 1993. 
[6] J. E. Baran and D. A. Smith; "Adiabatic $2 \times 2$ polarization splitter on $\mathrm{LiNbO}_{3}$ ', IEEE Photon. Technol. Lett., vol. 4, no. 1, pp. 39-40, 1992.

[7] T. Pohlman, A. Neyer and A. Voges: "Polarization independent $\mathrm{Ti}: \mathrm{LiNnO}_{3}$ switches and filters," IEEE J. of Quantum Electron., 27, pp. 602-607, 1991 .

[8] D. A. Smith, J. E. Baran, K. W. Cheung, and J. J. Johnson: "Polarizationindependent acoustically tunable optical filter," Appl. Phys. Lett., vol. 56, pp. 209-211, 1990.

[9] A. d'Alessandro, D. A. Smith and J. E. Baran: "Polarisation independent low power integrated acousto-optic tunable filter/switch using APE/Ti polarisation splitters on lithium niobate," Electron. Lett., vol. 20, pp. 1767-1769, 1993.

[10] D. A. Smith, A. d'Alessandro, J. E. Baran: "Reduction of crosstalk in an acousto-optic switch by means of dilation," accepted for publication in Opt. Lett., vol. 19, no. 2, pp. 99-101, 1994.

[11] D. A. Smith, A. d'Alessandro, J. E. Baran, H. Herrmann: "Source of sidelobe asymmetry in integrated acousto-optic filters," Appl. Phys. Lett., vol. 62 , pp. $814-816,1992$
[12] W. R. Trutna Jr., D. W. Dolfi, C. A. Flory: "Anoumalous sidelobes and birefringence apodization in acousto-optic tunable filters," Opt. Lett. vol. 18 , pp. $28-30,1993$.

[13] M. M. Choy, K. W. Cheung, D. A. Smith and J. E. Baran, "Observation of coherent interchannel interference in the multiwavelength operation of an acousto-optic filter," IEEE Photon. Technol. Lett., vol. 1, no. 7, p. $171,1989$.

[14] D. A. Smith and J. J. Johnson: "Sidelobe suppression in an acousto-optic filter with a raised-cosine in interaction strength," Appl. Phys. Lett., vol. 61 , pp. $1025-1027,1992$.

[15] H. Hermann and H. Schmid: "Integrated acousto-optical modeconvertors with weighted coupling using surface acoustic wave directional couplers," Electron. Lett., vol. 28, pp. 979-980, 1992.

[16] A. Kar-Roy and C. S. Tsai: "Low-sidelobe wighted-coupled integrated acoustooptic tunable filter using focused surface acoustic waves," Photon. Technol. Lett., vol. 4, pp. 1132-1135, 1992.

[17] Y. Yamamoto, C. S. Tsai and K. Esteghamat: "Guided-wave acoustooptic tunable filters using simple coupling weighting technique," IEEE Proc. Ultrasonics Symp. p. 605-608, 1990. 